\title{
Broncodilatadores en aerosol en la prevención del asma inducida por ejercicio
}

\author{
Carlos Aranda P. ${ }^{1}$; Ana María Merello M. ${ }^{2}$; Alan Power G. ${ }^{2}$; \\ Marcia Reus M. $^{2}$; Pedro Astudillo O. $^{3}$
}

\section{Prevention of exercise induced asthma by bronchodilator drug aerosol}

Four pressurized spray bronchodilator drug praparations and placebo were compared in their ability to inhibit exercise induced asthma in 16 asthmatic children ( 4 to 12 vears old), by measures of the maximum expiratory flow index fall after free running exercise test. Average flow index fall was 1.96 for fenoterol; 15.31 for ipratropium bromide; 5.21 for salbutamol and -0.47 for the mix ture of fenoterol and ipratropium bromide. Thus all four drug preparations were able to inhibit exercise-induced asthma but not placebo (beclomethasone, maan indax fall $24.72 \mathrm{~h}$. The efficacy of ipratropium bromide as a single drug was significantly lower than that of the other preparat ions.

iKay words: Asthma, exercise induced, aerosal therapy, prevention albuterol, fenoterol, ipratropium.\}

1. Unidad de Broncopulmonar, Servicio de Pediatría, Hospital Félix Bulnes.

2. Kinesiólogo. Servicio de Kinesiología, Hospital Féllx Bulines.

3. Instituto de Enfermedades Respiratorias Infantiles.
Los fármacos en aerosol envasados a presión (aerosoles) constituyen actualmente los medicamentos de elección en el tratamiento del asma ${ }^{1}$. En nuestro pais se han introducido nuevas combinaciones de medicamentos respecto de los 
cuales se hace necesario disponer de evidencias clínicas y de laboratorio que avalen su prescripción, por cuanto la indicación de un tratamiento requiere ponderar tanto la eficacia clínica como los costos de las drogas.

El objetivo del presente estudio fue evaluar la capacidad para inhibir el asma inducida por ejercicio (AIE), de los siguientes broncodilatadores en aerosol y envase presurizado: fenoterol (F), salbutamol (S), bromuro de ipratropio (BI) y la combinación de bromuro de ipratropio más fenoteral $(\mathrm{F}+\mathrm{BI})$.

\section{Material y Método}

Este estudio se realizó en 16 escolares ( 3 de sexo femenizo), de 4 a 12 ax̃os de edad (promedio 8,4 años), promedio de talla $131 \mathrm{~cm}$ (márgenes 109 y $145 \mathrm{~cm}$ ), controlados en nuestro servicio de enfermedades respiratotias, que tenían antecedentes clinicos de AJE documentada mediante prueba de ejercicio, consistente en carrera libre de 6 minutos de duración y medición de flujo espiratorio máximo (PEF), con flujómetro de $W_{r i g h t}{ }^{2}$, antes del ejercicio y en $\operatorname{los} 3,5,10,20$ y 30 minutos posteriores al término de la carrera. En todos los niños se registraron índices de caida (IC) ${ }^{3}$ del PEF mayores que $12 \% 4$. Los índices de caida fueron calculados camo el porcentaje de disminución del PEF producido por el ejercicio, con respecto al valor basal.
Tres dias antes del estudio se suspendió el uso de los medicamentos broncodilatadores. A cada niño se realizaron pruebas de ejercicio iguales a la descrita, en dias consecutivos y en similares condiciones ambientales, usando un método de toble clego. Veinte minutos antes del inicio de las pruebas, cada niño recibió, con una secuencia aleatoria, međiante espaciador o aerocáma$\mathbf{r a}^{5}$, dos descargas de uno de los siguientes medicamentos, en sus dosis habituales: fenoterol $200 \mu \mathrm{g}$ por descarga; salbutamol $100 \mu \mathrm{g}$ por descarga; bromuro de ipratropio $200 \mu \mathrm{g}$ por descarga; fenoterol más bromuro de ipratropio 50 y $200 \mu \mathrm{g}$ por descarga, respectivamente, y dipropionato de beclometasona $50 \mathrm{\mu g}$ par descarga, en calidad de placebo (P)* Los resultados se analizaron según el grado de protección del AIE que indujo cada đroga, usando como paránetro el IC. El estudio estadístico se reatizó sometiendo el IC obtenido con cada medicamento, a un análisis de varianza. Se aplícó la dócima de Student Neumann Keuls (SNK) ${ }^{\gamma}$ para valorar las diferencias entre las diversas drogas. Se usó una probabilidad de $0,05 \%$ como límite de diferenciación para el análisis de vatianza y de $0,01 \%$ para la comparación entre los medicamentos.

\section{Resultados}

Los flujos espiratorios iniciales de todos los pacientes, como porcentaje del valor teórico, en los diferentes dias del estudio se muestran en la tabla 1.

Tabla 1

PEF* injeiales de los pacientes estudiados

(como porcentaje del valor teórico para cada prueba)

\begin{tabular}{rrrrrr}
\hline Paciente & P & F & F+BI & S & BI \\
\hline 1 & 88 & 71 & 75 & 71 & 84 \\
2 & 87 & 83 & 94 & 83 & 83 \\
3 & 72 & 77 & 77 & 77 & 77 \\
4 & 86 & 94 & 91 & 102 & 94 \\
5 & 110 & 105 & 100 & 90 & 80 \\
6 & 92 & 92 & 86 & 95 & 80 \\
7 & 82 & 91 & 91 & 91 & 97 \\
8 & 90 & 90 & 92 & 88 & 90 \\
9 & 104 & 110 & 104 & 113 & 95 \\
10 & 89 & 95 & 92 & 83 & 95 \\
11 & 104 & 101 & 106 & 95 & 112 \\
12 & 95 & 116 & 98 & 112 & 105 \\
13 & 117 & 98 & 95 & 105 & 91 \\
14 & 109 & 95 & 99 & 82 & 96 \\
15 & 91 & 93 & 96 & 92 & 92 \\
16 & 93 & 95 & 11 & 12 & 11 \\
\hline Promedio & 94 & 11 & 11,45 & 13,04 & 11,95 \\
DE & 11 & 11,57 & & 95 \\
CV & 11,70 & & & 95 \\
\hline
\end{tabular}

*: en 1/min. DE: desviación estandar. CV: coeficiente de variación (11). 
Los promedios de IC (tabla 2) para cada droga fueron para fenoterol $1,96 \pm 3,77$; bromuro de ipratropio $15,31 \pm 5,62$; salbutamol 5,21 $\pm 8,81$; fenoterol con bromuro de ipratropio $-0,47 \pm$ 12,99, y beclometasona (placebo) 24,72 $\pm 13,18$. El análisis de varianza mostró una probabilidad de $5,64 \times 10^{-10}$, lo cual expresa diferencias sig. nificativas entre un par de medicamentos por lo menos $(p<0,05)$.

La prueba de SNK (tabla 3) permitió establecer que todos los broncodilatadores usados bloquearon en forma significativa la aparición del AIE respecto del placebo $(p<0,01)$. Fenoterol con bromuro de ipratropio, salbutamol y fenoterol solos, proporcionaron resultados similares y sus minimas diferencias se deben probablemente al azar ( $p>0,01$ ). El bromuro de ipratropio aisladamente, si bien dio resultados superiores que el placebo, fue significativamente menos eficaz que los demás preparados de broncodilatadores $(\mathrm{p}<0,01)$.

\section{Comentario}

Nuestros resultados permiten afirmar que las cuatro drogas usadas previenen significativamente el AIE respecto del placebo. Al comparar las drogas entre sí, fenoterol solo o combinado con iprattopio, así como salbutamol, son equivalentes en su capacidad para inhibir este fenómeno, siendo mucho menos efectivo el ipratropio como único medicamento. Otros trabajos han mostrado similares resultados con anticolinérgicos, lo que se explica porque el bromuro de ipratropio alcanza su máxima acción más tarde que en el periodo de observación definido para este trabajo y porque es un broncodilatador menos potente que los agentes adrenérgicos ${ }^{9}$.

Tabla 2

Promedios de índice de caída para cada droga

\begin{tabular}{lrr}
\hline & \multicolumn{1}{c}{$\overline{\mathrm{x}}$} & \multicolumn{1}{c}{ DE } \\
\hline Placebo & 24,72 & $\mathbf{1 3 , 1 8}$ \\
Bromuro de ipratropio & 15,31 & 5,62 \\
Salbutamol & 5,21 & $\mathbf{8 , 8 1}$ \\
Fenoterol & 1,96 & $\mathbf{3 , 7 7}$ \\
Fenoterol + bromuro de ipratropio & $-0,47$ & 12,99 \\
\hline
\end{tabular}

Nos llama la atención la buena protección que otorgó la combinación de fenoterol e ipratropio contra el asma inducido por ejercicio, por cuanto la dosis de fenoterol de esta com. binación es sólo de $50 \mu \mathrm{g}$, como recién comentamos, el anticolinérgico no sería un buen inhibidor. La favorable respuesta obtenida podría deberse a la acción simultánea del efecto antivagal $y$ el inhibidor de los mediadores biológicos del proceso asmático, los que se potenciarían en sus efectos ${ }^{10}$.

\section{Resumen}

Se comparó el efecto de cuatro medicamentos broncodilatadores en aerosol a presión, en cuanto a su capacidad para inhibir el asma inducida por ejercicio (AIE) en 16 niños asmáticos de 4 a 12 años de edad, mediante cálculo del índice de caída del flujo espiratorio máximo después de prueba de ejercicio con carrera libre. Las cuatro drogas y sus respectivos promedios de índice de caída fueron fenoterol 1,96; bromuro de ipratropio 15,31; salbutamol 5,21 y la combinación de fenoteral más bromuro de ipratropio $-0,47$. Todas ellas fueron capaces de inhibir el asma inducida por ejercicio con respecto del placebo (beclometasona) cuyo índice promedio de caida de flujo fue 24,72 . La efjcacia del bromuro de ipratropio fue significativamente menor que la de las otras drogas estudiadas.

(Palabras chaves: Asma, inducida por ejercicio, prevención, broncodilatadores, beta miméticos, anticolinérgicos, beclometasona.)

Tabla 3

Comparación entre las drogas estudiadas

\begin{tabular}{lc} 
Beclometasona v. ipratropio & s \\
Beclometasona v. salbutamol & s \\
Beclometasona v. fenoterol & s \\
Beclometasona v. fenoterol/ipratropio & s \\
Ipratropio v. salbutamol & s \\
Ipratropio v. fenoterol & s \\
Ipratropio v. fenoterol/ipratropio & s \\
Salbutamol v. fenoterol & ns \\
Salbutamol v. fenoterol/ipratropio & ns \\
Fenoterol v. fenoterol/ipratropio & ns \\
\hline
\end{tabular}

s: significativo; ns: no significativo; $\quad$ SNK p: 0,01 


\section{Referencias}

1. Godfrey $S, K \delta$ nig $P$ : Suppression of exercise-induced asthma by salbutamol, theophylline, atropine, cromolyn and placebo in a group of asthmatic children. Pediatrics 1975; 56 (suppl): 930-934.

2. Wright $B$, McKerrow $C$ : Maximum forced expiratory flow rate as a measure of ventilatory capacity. with a description of a new portable instrument for measuring it. Br Med Ir 1959; 2: 104l-1046.

3. Jiménez J. Cesar C, Díaz A. Cerutti E: Broncoconstricción inducida por ejercicio en niños asmáticos y nomales. Pediatría (Santiago) 1981; $24: 3-7$.

4. Taussig LM, Chernick V, Mellins RB: Standardization lung function testing in children. J Pediatr 1980;97:668-676.

5. Aronda C, Power A, Merello AM, Parra A: Cámara aérea para medicamentos presurizados. Rev Chil Pediatr 1986; $57: 152-156$.
6. König $P$, Jaffe, $P$, Godfrey $S$ : Effect of corticosteroids on exercise-induced asthma. J Allergy Clin Immunol 1974; \$4: 14-18.

7. Schefler W: Bioestadística $2^{\mathrm{a}}$ Ed. Fondo Educativo Interamericano S.A. México, 1981.

8. Ruffin RE, Fitzgerald JD, Rebuck AS: A comparison of the bronchodilator activity of SCH 1000 and salbutamol. J Allergy Clin Immunol 1977; 59: $136 \cdot 141$.

9. Gross $N$ : Ipratropium Bromide. N Engl J Med $1988 ; 319 ; 486494$.

10. Boushey HA: Pruebas de provocación bronquial. Enfermedades Respiratorias, rol del laboratorio. I.M.C. Ediciones Científicas, Santiago, Chile, $1986 ; 5: 65-76$.

11. Sivermon $M$, Anderson $S$ : Standardization of exercise tests in asthmatic children. Arch Dis Child $1972 ; 47: 882-889$. 